\title{
Some remarks on the use of avian malaria parasites in research
}

\author{
par Augusto CORRADETTI
}

Istituto Superiore di Sanità, Rome, Italie

\begin{abstract}
Résumé
L'utilisation des plasmodies aviaires est recommandée pour l'étude des problèmes concernant le paludisme humain. Des «modèles» de recherches avec des parasites aviaires sont proposés qui pourraient être appliqués à des études variées de chimiothérapie, pathologie, immunologie et biologie du paludisme.
\end{abstract}

\section{Summary}

The employment of avian plasmodia is emphasized for solving problems related to human malaria. Models of research with avian malaria parasites are reported, which can be applied to various studies of chemotherapeutics, pathology, immunology and biology of malaria.

The achievements obtained in investigations upon avian malaria parasites have been extensively summarized by Huff in a monograph published in 1963 in " Advances in Parasitology », and by Garnham (1966) in his magnificent book « Malaria Parasites and other Haemosporidia». Both these sources of reference are easily available and I will not repeat the same matter here. 
I will consequently limit the objective of the present paper to calling attention on the principal lines along which research on avian malaria can be carried out to obtain results of general interest or to solve problems arising from human malaria.

Attention of public health is obviously focused on human malaria : but human malaria is just a small part of the much larger picture constituted by Plasmodium infections as a whole. It is evident that a comparative study of each part of the picture will bring much ligth and will be essential for the correct understanding of human malaria.

The study of avian malaria is particularly interesting, because it presents a notable range of models of infection, some of which are deeply diverging among themselves and also from those observable in human malaria. This great variety of models permits very often the choice of a most suitable infection for investigations on some specific points.

To give an example of what has now been said, we can quote the studies on the mechanism of action of schizontocidal drugs. These studies usually need, for an easy and right interpretation, a parasite showing a high synchronicity of the schizogonic development in red cells. Plasmodium matutinum, $P$. giovannolai and $P$. cathemerium all meet this requirement.

Another field in which experiments with avian malaria is the method of choice, is for studies on the action of drugs destroying or sterilizing gametocytes. The best species for testing these types of drugs are $P$. gallinaceum and $P$. relictum: in fact these two parasites readily infect a high percentage of Aedes or Culex respectively. Consequently an eventual sterilizing action on the gametocytes is easily detected if the mosquitos remain uninfected after feeding on the bird to which the drug was administered.

Still remaining in the field of chemotherapy, light on some gaps observable in our knowledge of human malaria can be brought by the study of a very different model of infection occurring in avian malaria. For instance testing some schizontocidal drugs on $P$. elongatum revealed that the endo-haemoblastic cycle of the parasite was not affected by the drugs while the parasites living in mature red cells were killed. This phenomenon obviously depended on the fact that the drugs were in some way attached to the mature haemoglobin. This result with $P$. elongatum helps to understand why the same drugs do not show a totally suppressive action on $P$. berghei which generally lives in cells in which haemoglobin is at the pro-haemoglobin stage. The same result could also contribute to give an explanation of the higher frequency of relapses after treatment in $P$. vivax as compared to $P$. falciparum infections: as $P$. vivax starts its schizogonic life in reticulocytes, while $P$. falciparum starts in mature erythrocytes, there seems to be a higher probability for the first to escape the action of schizontocidal drugs.

The pathological patterns of infection in avian malaria are also differing essentially from those of human malaria.

For instance the development of sporozoites in avian malaria takes place in macrophages, Kuppfer cells, and other cells of the reticulo-endothelial system, while 
the sporozoites of the human plasmodia develop in hepatic parenchyma cells. Moreover in some avian malaria infections a late exo-erythrocytic cycle in reticulo-endothelial cells may take place, which is absent in human malaria. Here again, basic studies on the physiology and biochemistry of the parasites living in cells differing from red cells are easily established with avian parasites through the means of tissue culture and development in embryos, while they are not yet feasible with human Plasmodia.

Comparative studies on immunology in avian malaria are likely to give results of great value for enlightenment on the development of the immune response in general. As said before, avian Plasmodia show a variety of pathological courses of infection and it is natural that for each type of pathological course there is a corresponding type of immunity response. Thus different models of « pathological course/ immunity response * can be investigated in a single experimental host (Serinus canarius for passerine and Gallus gallus for gallinaceous Plasmodia), and everybody will agree on the advantages given by the employment of this material.

In some cases the models concerning a single immunity phenomenon can be much easier studied in bird than in human malaria. For instance the immunity crisis which takes place at the end of the primary attack is better followed quantitatively in birds in which an enormous number of basophilic erythroblasts appears in the blood circulation, than in man in which the response is given through the appearance of less easily detectable reticulocytes.

The low specificity shown by many avian Plasmodia for both the vertebrate and invertebrate hosts renders these parasites the material of choice for laboratory models intended to investigate processes of speciation, the selection of strains and possibly the captation of mutants in nature. As it is easily understood, this is an immense field to be explored, which is likely to give us a great amount of fundamental knowledge for the understanding of many factors involved in the epidemiology, zoogeography and ecology of human malaria.

The examples could be easily multiplied, but I believe that this very limited list is sufficient to give an idea of the extent to which avian malaria can be employed for fundamental research and can help to solve problems related to human malaria.

\section{References}

Garnham (P. C. C.), 1966. - «Malaria Parasites and other Haemosporidia », Oxford.

HufF (C. G.), 1963. - In «Advances in Parasitology», Edit. Ben Dawes, London and New York. 